\title{
Política 2.0 en Ecuador. Análisis del discurso y la comunicación política en Facebook
}

\section{Policy 2.0 in Ecuador. Analysis of discourse and political commu- nication on Facebook}

\author{
Verónica Altamirano - Benítez. \\ Universidad Técnica Particular de Loja. Ecuador. \\ vpaltamirano@utpl.edu.ec

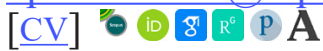 \\ Pablo Ruíz - Aguirre. \\ Universidad Internacional del Ecuador. Ecuador \\ paruizag@uide.edu.ec \\ (1) $8 \mathrm{R}^{\circ}$ \\ Gabriela Baquerizo-Neira. \\ Universidad Casa Grande. Ecuador. \\ gbaquerizo@casagrande.edu.ec \\ (1) (1) $\mathrm{R}^{\mathrm{P}} \mathrm{P}$
}

\begin{abstract}
Cómo citar este artículo / Referencia normalizada
Altamirano - Benítez, V., Ruíz - Aguirre, P. y Baquerizo-Neira, G. (2022). Política 2.0 en Ecuador. Análisis del discurso y la comunicación política en Facebook. Revista Latina de Comunicación Social, 80, 201-223. https://www.doi.org/10.4185/RLCS-2022-1539
\end{abstract}

\section{RESUMEN.}

Introducción: Las redes sociales son reconocidas por el impacto en las decisiones de los usuarios, y han sido estudiadas para determinar su incidencia en el ámbito político. Metodología: La presente investigación analiza la campaña electoral de los candidatos a presidentes de Ecuador, en el año 2021, a partir de tres objetivos: analizar la comunicación 2.0 para determinar en qué medida los candidatos establecen una relación interactiva con los electores en Facebook; analizar los elementos lingüísticos y las emociones en las publicaciones para identificar el tipo de discurso político que emplean; y determinar la agenda política digital y la oferta de campaña en las publicaciones realizadas por los presidenciables. Para alcanzar estos objetivos se implementó una metodología mixta. La investigación se desarrolló en dos etapas. En la primera etapa se aplicó el Modelo de evaluación de la comunicación 2.0 en las redes sociales, que desarrolla un análisis cualitativo, de igual forma se analizó y cuantificó somáticamente las emociones que se transmitieron en las campañas electorales y en la segunda etapa se codificó 1.543 publicaciones para identificar la oferta de campaña y la agenda política de los candidatos.Resultados: La comunicación política en las redes sociales no es estratégica, se observa un exceso de información que satura la red social y que además carece de contenido de valor para el ciudadano. Conclusiones: La comunicación política 2.0 es incipiente en Ecuador, se utiliza Facebook como un canal de comunicación tradicional, con contenido retórico, poco interesante, pero cargado de emocionalidad positiva para intentar conectar con el electorado.

PALABRAS CLAVE: Comunicación digital; política; campaña electoral; redes sociales; emociones; agenda política; discurso político.

\begin{abstract}
Introduction: Social networks are recognized for their impact on users' decisions. They have been studied to determine their incidence in the political arena. Methodology: This research analyzes the electoral campaign of the candidates for president of Ecuador, in the year 2021, based on three objectives: to analyze the 2.0 communication to determine to what extent the candidates establish an interactive
\end{abstract}


relationship with voters on Facebook; to analyze the linguistic elements and emotions in the publications to identify the type of political discourse they use; and to determine the digital political agenda in the publications elaborated by the presidential candidates, for their campaign offer. To achieve these objectives, a mixed methodology was implemented. The research was developed in two stages. In the first stage was applied the Model of evaluation of communication 2.0 in social networks, which develops a qualitative analysis, in the same way the emotions that were transmitted in the electoral campaigns were analyzed and quantified somatically, and in the second stage 1,543 publications were codified to identify the campaign offer and the political agenda of the candidates. Results: Political communication in social networks is not strategic, there is an excess of information that saturates the social network and also lacks content of value for the citizen. Conclusions: Political communication 2.0 is incipient in Ecuador, Facebook is used as a traditional communication channel, with rhetorical and uninteresting content, but loaded with positive emotionality to try to connect with the electorate.

KEYWORDS: Digital communication; politics; electoral campaign; social media; emotions; political agenda; political speech.

\section{CONTENIDO}

1. Introducción. 2. Objetivos. 3. Metodología. 4. Discusión/Resultados. 5. Conclusiones 6. Bibliografía 8. Currículum Vitae

\section{CONTENT}

1. Introduction. 2. Objectives. 3. Methodology. 4. Discussion / Results. 5. Conclusions. 6. Bibliography 8. Curriculum Vitae

\section{Introducción}

La comunicación política se encuentra en un proceso evolutivo, que inicia con una comunicación masiva y controlada, destinada a informar de manera asimétrica y unidireccional, a una comunicación multimediática en la Red. La característica principal de este nuevo modelo de comunicación es la participación activa y voluntaria de los electores a través de los entornos y plataformas digitales. Desde siempre, para decidir en el proceso electoral, los ciudadanos buscan información y referencias de los partidos políticos, especialmente, de los candidatos y sus propuestas de campaña, pero, este proceso de comunicación, en la actualidad, está influenciado por una actitud colaborativa del electorado, que implica comunicación bidireccional e interactiva desarrollándose de manera horizontal, en la que el ciudadano adquiere poder en el proceso de comunicación. Túñez y Altamirano (2015) afirman:

Es un hecho que la democratización de la posibilidad de ser emisor de mensajes con potenciales públicos de masas como receptores y la universalización de la conexión interpersonal a través de Internet permite a los usuarios interactuar de uno a uno, o de uno a varios o, como mucho, en pequeños grupos, y relacionarnos todos con todos. Han variado, también, los flujos del mensaje, evolucionando de un modo unidireccional (discursivo) a una circulación realmente interactiva y, en simultáneo, en varios soportes (p. 14)

"Las redes sociales se han mostrado como un entorno único por su posibilidad de conectar usuarios y facilitar la interacción entre la esfera política y el público" (Blasco-Duatis et al., 2018, p. 270). Es decir, la comunicación política evoluciona a un modelo bidireccional, participativo, voluntario y comprometido, en el que el ciudadano es quien controla los procesos de comunicación. En este escenario, "las redes sociales ofrecen mayores facilidades para contactar directamente, otorgar visibilidad a los participantes, fomentar los debates o incluso alertar sobre posibles conflictos" (Barandiarán, Unceta, y Peña, 2020, p.272).

Por tanto, al emplear estrategias de comunicación 2.0 es indispensable mantener una actitud y compromiso 2.0, es decir, adaptarse a las necesidades del usuario, que en la era digital es quien marca el rumbo y las tendencias. En este sentido, Nopo (2021) afirma que "las nuevas tecnologías están brindando un nuevo espacio público. Por un lado, las nuevas tecnologías ayudan a que los ciudadanos obtengan información de manera inmediata, y por otro, aperturan una ventana para que la ciudadanía ejerza su libertad de expresión" (p. 100). De esta manera, Facebook (FB) se consolida como un canal idóneo 
para la comunicación política. Es considerado un canal no "persuasivo" al basarse en tres principios como son la afiliación voluntaria de los usuarios, la aportación colaborativa de contenidos por parte de cada uno ellos y la atención voluntaria a las acciones que realizan las marcas (Alonso Mosquera y Muñoz de Luna, 2013), lo que permite desarrollar una comunicación no invasiva y directa con el público objetivo.

Para analizar la evolución de la comunicación política en los entornos digitales, la presente investigación plantea tres enfoques de estudio, primero se evalúa los procesos de comunicación 2.0 para determinar la interacción y la relación que se establece entre los candidatos y los electores a través de la red social Facebook.

En segundo lugar, se enfoca en el análisis del uso del lenguaje y emocionalidad del discurso de los candidatos, para ello, se aplica a los datos la herramienta de procesamiento de lenguaje natural, Linguistic Inquiry and Word Count (LIWC). En tercer lugar, se, busca comprender desde la perspectiva de la agenda política, por qué ciertos temas son abordados por los candidatos y otros no, con la finalidad de comprender en dónde está el foco de atención y cuáles son las prioridades de política pública que son escogidos en el discurso en las redes sociales. A partir del análisis de contenido, se examina el posicionamiento respecto los temas que más interesa transmitir a la ciudadanía a través de las propuestas electorales.

Con estos tres enfoques se procura realizar una triangulación metodológica y determinar qué elementos lingüísticos emplean en sus mensajes los líderes políticos ecuatorianos, la emocionalidad y los temas de la agenda política que prevalecen en sus posts. En definitiva, se determina cómo estos elementos lingüísticos también inciden en la comunicación 2.0 de los candidatos con sus seguidores.

\subsection{Facebook y política}

Las redes sociales aplicadas al ámbito político han tenido un auge en la última década. FB ha crecido de forma exponencial desde sus orígenes en febrero de 2004 llegando a posicionarse como la red con más seguidores a nivel mundial. Las principales características que presenta esta red para su éxito es la flexibilidad, la adaptación, la evolución y la capacidad viral que alcanzan los contenidos. Con estos antecedentes se debe considerar a FB como una herramienta de comunicación política y adaptarse al modelo de comunicación en el que el usuario es el centro y el contenido es el rey.

En el escenario político digital se modifican las relaciones de poder tradicionales en las que la hegemonía en la construcción del discurso la ostentaban las esferas política y mediática. En el contexto online, la sociedad civil, antes diana pasiva de los mensajes políticos mediatizados, adquiere un estatus similar al de las instituciones políticas o las empresas mediáticas, al posibilitar Internet y especialmente las plataformas sociales su participación directa y activa en los contenidos que circulan en la web, su agrupación y su autorregulación al margen de las élites discursivas (Gil Ramírez y Gómez de Travesedo Rojas, 2021, p. 65).

En FB los grupos creados por los consumidores o las organizaciones políticas proporcionan el foro idóneo para que pequeñas comunidades de personas con un interés común puedan comunicarse y expresarse en relación con dicho interés. Acercarse al discurso de las redes sociales, en el caso de Facebook, permite explorar nuevos relatos de participación política a partir del acceso directo que los ciudadanos tienen hacia los mensajes que ofrecen los diferentes partidos políticos, los políticos u otros medios de comunicación tradicionales vinculados al mundo digital (Rodríguez Fidalgo et al., 2017, p. 851). A pesar de que, generalmente, los usuarios de redes sociales no prestan especial atención a la información que proporcionan los perfiles de los partidos políticos y sus líderes no suelen ser particularmente populares, sus páginas de Facebook pueden atraer gran atención en momentos de mayor movilización y entusiasmo cívico, como ocurre durante los procesos electorales (Citado en Valera-Ordaz et al., 2018, p. 57).

El punto de partida del uso de FB en política fue la campaña presidencial de Barak Obama, en el año 2008, en la que se "creó una organización virtual a nivel nacional que motivó a 3,1 millones de contribuyentes individuales y movilizó un movimiento de base de más de 5 millones de voluntarios" (Cogburn y Espinoza-Vásquez, 2011, p.189), lo que permitió, no solo segmentar los públicos y recau- 
dar fondos, sino, ganar las elecciones. La situación fue similar en la campaña de Trump del 2016 en Estados Unidos, la inversión en nuevas plataformas y redes sociales logró que domine el panorama mediático.

Al ser FB una red social utilizada por 2.740 millones de personas diariamente (Galeano, 2021), es una plataforma que permite interactuar con los votantes, especialmente con los jóvenes. Sweetser y Lariscy (2008), tras estudiar 5.735 comentarios de FB de los candidatos a la Cámara de Representantes y al Senado de Estados Unidos pudieron observar, que los mensajes de los ciudadanos con los candidatos eran positivos, pero sin contenido, superficiales. Sin embargo, la comunicación fue simétrica y bidireccional, lo que provocó una relación "amistosa" entre el candidato y el votante. Por su parte, Bode et al. (2014) confirman que se puede predecir la participación política de los adolescentes -segmento del electorado con una tradición poco activa en estos temas- a partir del uso que estos hacen de las redes sociales, "especialmente si se les puede animar a ser voluntarios, contribuir y hacer proselitismo entre sus amigos y compañeros de Facebook" (Williams y Gulati, 2009, p.2). Una revisión de literatura realizada por Eric Van Steenburg (2015) también determina que "la investigación sobre la publicidad política está floreciendo", y una de las vías es la "publicidad basada en la resonancia y la emoción" (p.30).

Existen otros estudios sobre el uso político de FB que se centran en el aumento de la conciencia política (Pasek et al., 2006; Vitak et al., 2011); en la configuración de la toma de decisiones políticas (Kushin y Yamamoto, 2013); en el impacto del uso político de FB (Bode et al., 2014; Vitak et al., 2011), entre otros. Estudios locales como el realizado por Baquerizo-Neira et al. (2021) analizan el uso del lenguaje y los temas promovidos por las candidatas a las alcaldías de Guayaquil y Quito en las elecciones municipales de marzo de 2019. Aplican la técnica de análisis de contenido digital y la herramienta de monitorización Fanpage Karma a 743 publicaciones y como resultado demuestran que FB se emplea principalmente como altavoz de la campaña offline, como un espacio de promoción de las propuestas políticas y no como una plataforma de interacción entre las mujeres políticas y sus seguidores.

\subsection{Análisis de sentimiento y redes sociales}

La herramienta de procesamiento de lenguaje natural LIWC fue diseñada para, a partir de la escritura, identificar "experiencias vitales negativas que podían predecir mejoras posteriores en la salud", se ha utilizado ampliamente en psicología y lingüística (Tausczik y Pennebaker, 2009). Actualmente, también se utiliza para examinar una amplia variedad de fuentes textuales como: fragmentos literarios, piezas publicitarias, narraciones personales, conferencias, libros de texto, conversaciones, etc. (Pennebaker et al., 2003, p. 553). Por ejemplo, Newman et al., (2008) lo emplean para examinar las diferencias de género en el uso del lenguaje, esto lo hicieron mediante el análisis de una base de datos de más de 14.000 archivos de texto procedentes de 70 estudios distintos.

Otros autores como, Deltell et al. (2013) lo aplican para predecir la tendencia política de las elecciones andaluzas 2012. Estos expertos indagan en el flujo y contenido de los tweets para determinar si se podría predecir el "sentimiento y las tendencias políticas dentro de una comunidad" mediante la caracterización de la personalidad de los usuarios en las redes sociales.

Alashri et al. (2018) realizan el análisis de sentimiento de 22 mil publicaciones de candidatos políticos en FB y más de 48 millones de comentarios, para determinar la interacción entre estos y los ciudadanos durante las elecciones presidenciales en Estados Unidos del 2016. Como resultado clasifican a los participantes en función de los sentimientos positivos y negativos identificados en sus comentarios, además de las diferencias psicológicas y lingüísticas que se pueden observar en los mensajes de los usuarios analizados.

En redes sociales también lo han aplicado Park et al. (2015), que analizan las publicaciones de FB de 66.732 usuarios para predecir su personalidad. En cambio, Dutt et al. (2018) realizan el análisis de 3.500 anuncios publicitarios de FB utilizando técnicas de procesamiento del lenguaje natural, con la finalidad de identificar las características textuales y semánticas asociadas a los anuncios publicitarios más efectivos. Es así como se descubre que la publicidad tiene un sentimiento menos positivo y está centrada en situaciones pasadas. Además, hallan que la duración de las campañas y la promoción de los anuncios no buscan influir en las elecciones. 
En investigaciones locales, Pita et al., (2016) analizan en la plataforma de microblogging Twitter los perfiles lingüísticos de líderes políticos ecuatorianos. Para ello aplican la herramienta LIWC a 330.000 tuits recogidos de 221 tuiteros considerados como: líderes políticos, seguidores de líderes y usuarios comunes. Construyen un vector para los tuits de cada usuario empleando 12 dimensiones psicológicas del software LIWC y comparan los usuarios con diferentes perfiles. Como hallazgos, estos expertos encuentran que el perfil del grupo de líderes es diferente y que alrededor del $30 \%$ de los seguidores de los líderes son similares a, por lo menos, un líder.

\subsection{Agenda política en los medios sociales}

La literatura académica establece que los partidos políticos intentan aprovechar la agenda política (los temas más importantes sobre los que se debate) en aquellos temas en los que deben ser dignos de confianza (Budge y Hofferbert 1990; Klingemann et al., 1994). Esto refiere, que un partido político habla de economía, de salud pública o de administración si sobre esas temáticas tiene experiencia o ha marcado agenda antes. De esta manera, la competencia electoral en un sistema democrático consiste en enfatizar ciertas cuestiones y anular o silenciar otras (Cruz et al., 2020). En esta línea, una gran cantidad de estudios en la comunidad del "Comparative policy agendas" partiendo de la teoría de la "agenda setting" o establecimiento de la agenda, utilizan la teoría propiedad del tema, para exponer por qué las élites políticas prestan atención a algunos temas y no a otros (Green y Jennings 2012; Green y Jennings, 2019; Green - Pedersen y Mortensen, 2010).

Esta teoría se desarrolló, originalmente, para explicar por qué los partidos prestan atención a temas particulares durante las campañas electorales. En este sentido, se considera que los partidos políticos destacan asuntos en los que tienen una reputación de mayor competencia y sus oponentes son menos considerados (Budge y Farlie, 1983; Petrocik, 1996). En esta línea, es de esperar que los partidos de izquierda se concentren más en temas relacionados con el bienestar, la igualdad de derechos o el medio ambiente, donde el público percibe que se desempeñan mejor y que acentúen sus promesas electorales, sus discursos en estas temáticas. Por otro lado, se espera que los partidos de derecha, por el contrario, presten más atención a temas como la economía, los impuestos o la delincuencia donde tienen una reputación de buen desempeño.

Los estudios de las agendas políticas de Inglaterra, sobre el discurso del trono, por ejemplo, determinan que el papel de establecimiento de la agenda del discurso refleja las fortalezas de los partidos políticos. Significa que, los gobiernos conservadores tienden a prestar menos atención a los derechos civiles y los problemas de las minorías, los problemas sociales y las operaciones gubernamentales, pero, históricamente, prestan más atención a los asuntos internacionales que otros gobiernos (Jennings et al., 2011).

Sin embargo, en los nuevos escenarios digitales donde la información política juega un papel determinante en la conformación de las agendas, y la especialización periodística afronta la complejidad de integrar el conocimiento con un nuevo modelo de producción de la información, condensado en cada vez menos espacio e integrado en las nuevas pautas de consumos de medios y de noticias (LópezLópez y Vásquez-González, 2018), se precisa analizar cómo los candidatos políticos abordan los temas de interés en los medios sociales. Siguiendo estas ideas, ¿podría esperarse que la agenda que se intenta marcar en campaña electoral sigue esta lógica partidista? ¿Los candidatos más cercanos a la izquierda como Pérez, Arauz; de social democracia como Hervas y de derecha como Lasso mantienen esta lógica? ¿Cuáles son los temas más importantes que resaltaron en campaña electoral a través de Facebook? ¿Existe emocionalidad positiva o negativa en sus publicaciones? Son algunas dudas que se intenta responder, entendiendo los posts como sus elementos discursivos.

\section{Objetivos}

La investigación analiza la campaña electoral de los candidatos a presidentes de Ecuador en las elecciones 2021 en FB, para lo cual se plantea tres objetivos:

1) Evaluar los procesos de comunicación 2.0 entre los candidatos a presidentes y los electores, partiendo de la premisa que la comunicación en los medios sociales debe ser bidireccional y fortalecer la relación horizontal.

2) Analizar los elementos lingüísticos y emocionalidad de las publicaciones de los candidatos a la pre- 
sidencia como recursos de su estrategia de comunicación en campaña.

3) Determinar la agenda política digital, cuáles fueron los temas que abordaron los candidatos en la red social.

\section{Metodología}

Para cumplir con los objetivos propuestos, se utiliza una metodología mixta, que consiste en la integración sistemática de los métodos cuantitativo y cualitativo, en un solo estudio, con el fin de obtener una visión completa del fenómeno abordado. Esta metodología permite "generar y verificar teorías, ampliar la confianza, validez y comprensión de los resultados" (Chaves - Montero, 2018, p. 182).

La investigación se realiza durante el periodo electoral 2021, que inicia el 31 de diciembre de 2020 y finaliza el 7 de febrero de 2021. En la primera etapa, se evalúan los procesos de comunicación seleccionando como muestra los 15 candidatos a la presidencia, con la finalidad de determinar si la implementación de estrategias digitales exitosas incide en la decisión de voto. En la segunda fase, para realizar el análisis de contenido en las cuentas oficiales de FB, se selecciona a los candidatos que obtienen el $87.52 \%$ de la votación. No se analiza a los postulantes que no superaran el 3\% de la aceptación de los ecuatorianos. En la investigación se codificaron 1.543 post, 408 publicaciones de Andrés Arauz, 449 post de Guillermo Lasso, 369 publicaciones de Yaku Pérez y 317 de Xavier Hervas.

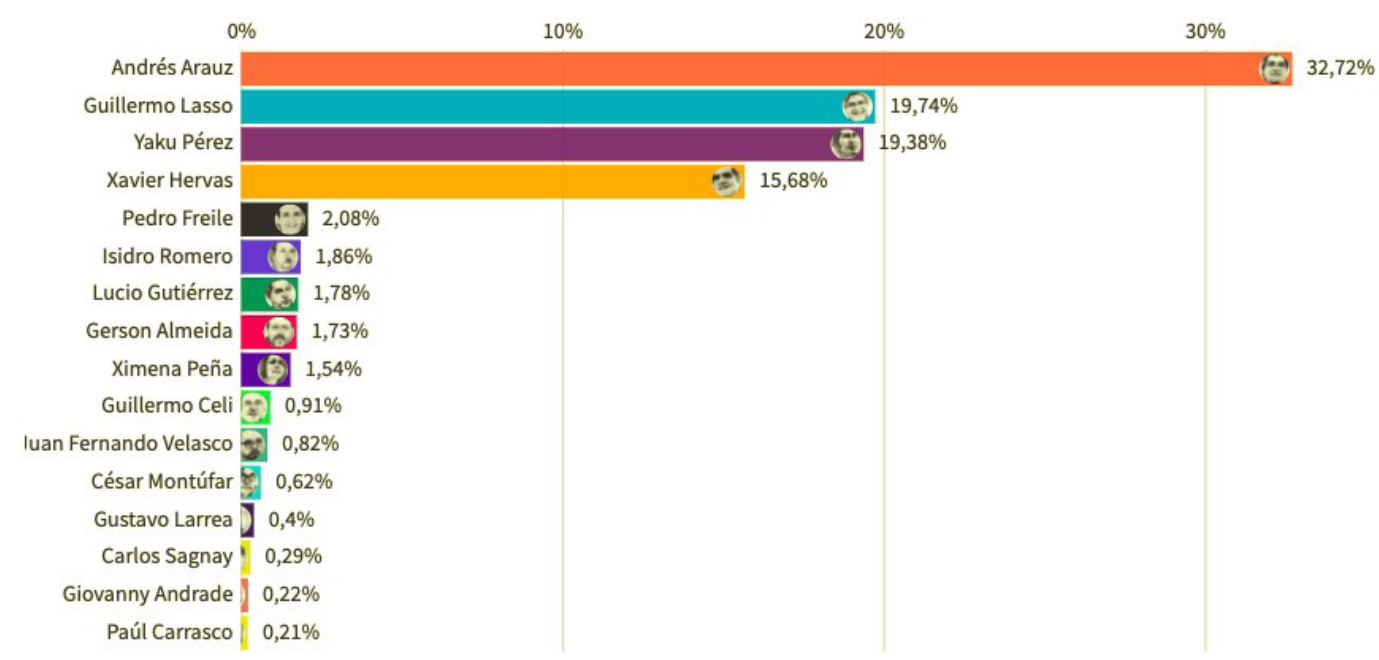

Gráfico 1: Resultados de la $1^{\mathrm{a}}$ vuelta electoral

Fuente: Primicias (2021). t.ly/ZxC3

\subsection{Analítica Social}

Una de las principales ventajas de los medios sociales es la cantidad de información que proporcionan de sus seguidores y su comportamiento en la red. Aunque no se llega a un consenso en cómo valorar los medios sociales, los autores concuerdan que dependiendo de las necesidades de la investigación se deben seleccionar los indicadores clave de rendimiento (Key Performance Indicator KPI). Los KPIs permiten evaluar los resultados de las campañas en las redes sociales.

Para realizar el análisis cuantitativo, se utilizó el Modelo de Evaluación de la Comunicación 2.0 en los Medios Sociales, que valora la comunicación 2.0 que se desarrolla en los medios sociales, partiendo de la premisa que este tipo de promoción debe cumplir con las características principales de la Web 2.0: La comunicación política 2.0, se basa en la interacción que se genera entre los candidatos y los electores 2.0. Para que se consiga esta interacción se requiere que los partidos políticos o los candidatos creen las condiciones adecuadas, por tanto, es necesario que se consolide una comunidad, que los candidatos actualicen permanentemente los contenidos y promuevan la participación de los usuarios a través de la co-creación de contenidos, el diálogo y la interacción con los seguidores estableciendo una comunicación bidireccional y horizontal en espacios de igualdad. Por otro lado, la participación y el compromiso de los usuarios son indispensables para el desarrollo de la comunicación 2.0. 
Para determinar los procesos de comunicación entre los candidatos presidenciales y el electorado se evalúan seis variables que interrelacionan los siguientes indicadores claves de desempeño: presencia, crecimiento, actividad, nivel de servicio, participación y engagement (Altamirano, et al., 2018).

\subsection{Análisis de Sentimiento}

Una vez que se realiza la extracción de FB, se obtiene el corpus para el análisis y se procesa cada post a través del programa Linguistic Inquiry and Word Count (LIWC) para determinar el uso del lenguaje de los candidatos en el contexto de la campaña electoral. Este software provee un diccionario en habla inglesa y española que está validado empíricamente para reflejar una dimensión psicológica en diferentes contextos (Pennebaker et al., 2003).

El programa analiza más de 70 variables lingüísticas. Se procesan en total 1.543 publicaciones de los candidatos a la presidencia (ver gráfico 2), a los que se les aplica todas las categorías proporcionadas por la herramienta, esto se realiza con el objetivo de identificar la emocionalidad como estrategia del discurso empleada en las publicaciones de los candidatos. Para cada dimensión psicológica el programa informático calcula la frecuencia relativa con que las palabras relacionadas con esa dimensión aparecen en un post.

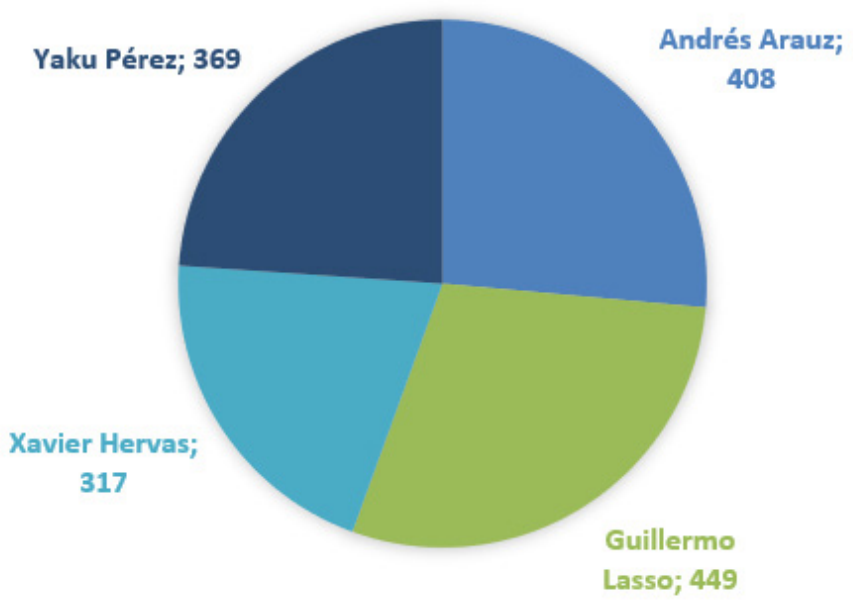

Gráfico 2. Publicaciones en Facebook Fuente: Elaborado por los autores

Con este análisis se observa que los candidatos políticos ecuatorianos emplean, en mayor medida, como recursos lingüísticos los pronombres personales (yo, nosotros, tu/usted, él/ella, ellos); procesos psicológicos: emociones positivas y negativas; $y$, dimensiones del contenido tradicional como temas sociales: familia, necesidades sociales, amigos, asuntos humanos. Y, otros temas como: tiempo, trabajo, logro, hogar, dinero, religión. Estos últimos se contrastaron con la agenda política de los candidatos.

\subsection{Análisis de contenido}

El análisis de contenido es una técnica para estudiar y analizar la comunicación de una manera objetiva, sistemática y cuantitativa. "En ocasiones se extiende la definición del análisis de contenido a una técnica de investigación para hacer inferencias válidas y confiables de datos con respecto a su contexto" (Toro Jaramillo y Parra Ramírez, 2006, p. 169).

Para estudiar la agenda política planteada a través de los posts de Facebook, se utiliza una la metodología usada por el Proyecto de Agendas de Política Ecuatoriana (Aguirre, 2020), proyecto de investigación aplicado a Ecuador. Esta codificación se realiza de acuerdo con el marco de codificación de contenido de políticas creado por Spanish Policy Agendas Project (https://q-dem.com/es/), para la relación lingüística, también basado en el marco de codificación de contenido de políticas originalmente creado por Baumgartner y Jones (2010), (https://www.comparativeagendas.net/) para investigar la configuración de la agenda en los EE.UU. Para este estudio se considera el libro de códigos adaptado 
para Ecuador basado en el libro de códigos maestro.

El sistema de codificación ecuatoriano involucra categorías para los principales temas de política pública (24) y diferentes subtemas dentro de cada una de estas categorías, 247 (ver Anexo 1). El proyecto creó una versión nacional del libro de códigos maestro, manteniendo las categorías originales, pero agregando un nuevo código (9) relacionado con el modelo de desarrollo "buen vivir" de Ecuador y algunos subcódigos de acuerdo con la realidad ecuatoriana. Se consideró una particularidad en el proceso de codificación, el código 0, se lo ha denominado "retórica". Es una unidad de análisis que no se pudo codificar de acuerdo con la metodología propuesta.

Es importante explicar la necesidad de incluir estos dos últimos códigos. En primer lugar, respecto el código 9, buen vivir. Se lo consideró tomando en cuenta que la filosofía político-ideológica del proceso político de la revolución ciudadana, el "buen vivir", se materializó en la Constitución de la república de 2008 a través de una asamblea constituyente. Dicha Constitución mantiene un marco normativo en donde dedica derechos alrededor de esta filosofía, "buen vivir", y ella no es posible codificar de acuerdo a la lista de códigos base, justamente por su epistemología occidental y anglosajona. Ante ello, el considerar este código permitió dar cabida a la consideración jurídica del país.

En segundo lugar, una de las consideraciones a través de los estudios de la agenda política discursiva, denota que, en América Latina es regular el discurso emocional, no lógico, y narrativo (Aguirre, 2016) que no apela a ideas claras, sino que mantiene el objetivo de convencer y persuadir. Considerando que la comunicación política del caso de estudio se circunscribe a esta forma de comunicar latina, se toma en cuenta este código no solo para medir cuando no hay agenda política, sino cuando se habla de manera distinta. Cabe aclarar que una posibilidad, sin duda sería no tomar en cuenta este código y medir únicamente el foco de atención de una manera tradicional, sin embargo, ello supondría no visibilizar la riqueza discursiva de la realidad del país, y occidentalizar el discurso.

Si bien este último código " 0 " no se menciona en los estudios de establecimiento de la agenda, se ha mantenido aquí por dos razones. Primero, como resultado de la codificación había temas a nivel de las cuasi-oraciones, considerando los posts de FB, que no estaban sujetos a ninguna codificación porque no tenían contenido de política. En estas unidades de análisis los candidatos contaban una anécdota, agradecían ciertas cuestiones o usaban frases de contenido emocional, así como usaban frases slogans propios de sus campañas electorales. Segundo, porque este código es el que tiene mayor porcentaje de atención entre todos los posts analizados. Es decir, los candidatos les dan más atención a cuestiones "retóricas" que otras temáticas de contenido político, incluso de aquellos que han sido de mayor importancia, como gobierno, economía o derechos y libertades.

Los posts se han codificado a nivel de cuasi oraciones. Una cuasi-oración (o declaración de política) es una manifestación de una sola idea o tema de política, pero no es esencialmente una oración completa (Volkens, 2002). Por lo general, este componente de análisis es identificable por el uso del punto final de la oración dentro de un post. Las transcripciones de las publicaciones fueron codificadas a ciegas por tres investigadores, primero para determinar si cada cuasi-oración tenía algún contenido de política, y segundo para asignar un código de tema principal y un código de subtema a la cuasi-oración. Este sistema dio lugar a un acuerdo entre codificadores del $85 \%$ en la mayoría de los posts. Los codificadores resolvieron las diferencias residuales a través de un debate y el líder del proyecto tomó la decisión final cuando no se pudo acordar entre los codificadores.

Para comprobar si las publicaciones de los candidatos se enfocan en temas específicos como los derechos o si se enfocan en temas relacionados con las funciones tradicionales del Estado, es necesario medir cómo se fragmenta la atención en cada post y en la información agregada. Como resultado introductorio se obtiene el porcentaje de atención para cada tema, tomando en cuenta el valor de la frecuencia de cada tema con respecto a todas las unidades de análisis.

\section{Resultados y Discusión}

\subsection{Comunicación 2.0}

La comunicación política 2.0 se determina por la interacción y relación que se establece en FB entre los candidatos políticos y los electores, esta relación se fundamenta en una comunicación que se desa- 
rrolla en espacios de igualdad, por tanto, no debe ser tradicional o unidireccional, en la que el candidato habla desde una tarima virtual y el ciudadano escucha, por el contrario, el ciudadano tiene voz y el candidato establece espacios de diálogo con ellos.

\subsubsection{Presencia}

En la investigación se determina que existe relación entre los candidatos con mayor votación y los que tienen mayor presencia en la red, aunque no en el mismo orden. La presencia de Guillermo Lasso es de 1.046.416, el segundo candidato con mayor presencia es Juan Fernando Velasco con 278.750, es importante recalcar que es un cantante reconocido del país y durante la campaña utilizó su cuenta artística, le sigue Yaku Pérez (267.969), Xavier Hervas (143.956) y Andrés Arauz (136.070). Sin embargo, la penetración de los candidatos es baja, Lasso, alcanza el 8\% de los 13.3 millones de usuarios de la red en el país, (Del Alcázar Ponce, 2021) Velasco y Pérez consigue atraer al 2\% y Hervas y Arauz alcanzan el $1 \%$, los otros candidatos no superan el $0.4 \%$, que tiene relación con el porcentaje de votación que alcanzaron.

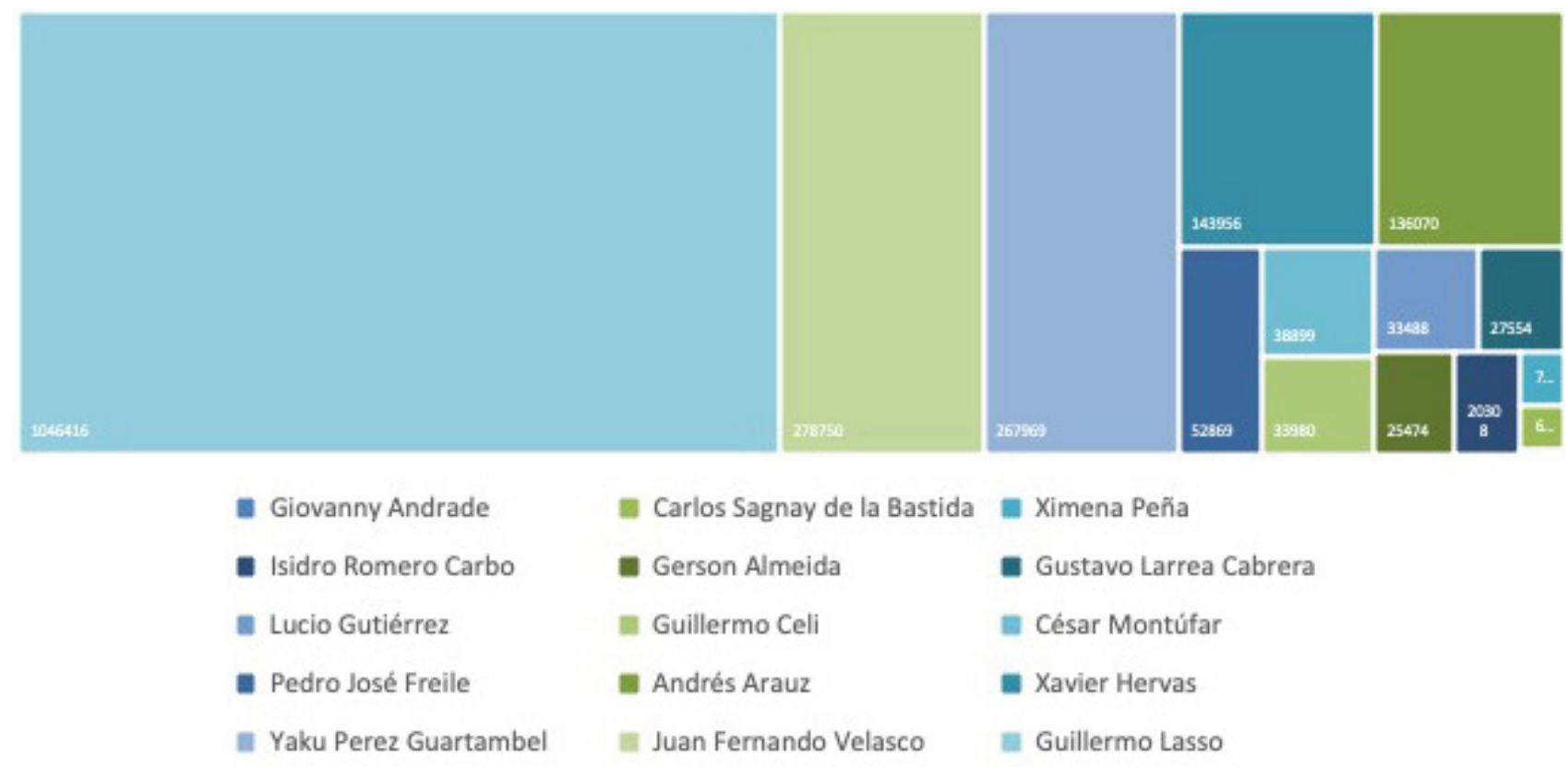

Gráfico 3: Presencia en Facebook

Fuente: Elaborado por los autores

\subsubsection{Crecimiento}

En cuanto al crecimiento destaca Pedro José Freire, que incorpora 30.087 fans, Yaku Pérez 15.631, Isidro Romero Carbo 11.973. Los otros candidatos no superan los 5000 seguidores nuevos, y 4 candidatos no generan crecimiento y en el caso de Gerson Almeida, pierde 384 fans, probablemente por la falta de estrategia digital y la saturación de la red social con información poco interesante.

\subsubsection{Actividad}

De la muestra analizada, se observa que Guillermo Lasso es el candidato más activo, publica en promedio 5 posts al día, mientras que 6 candidatos publican menos de un post diario, lo que no es estratégico en una campaña electoral porque saturan la red social con información del candidato como en el primer caso, o existe carencia de información y presencia como sucede con los candidatos Almeida, Andrade y Velasco. 


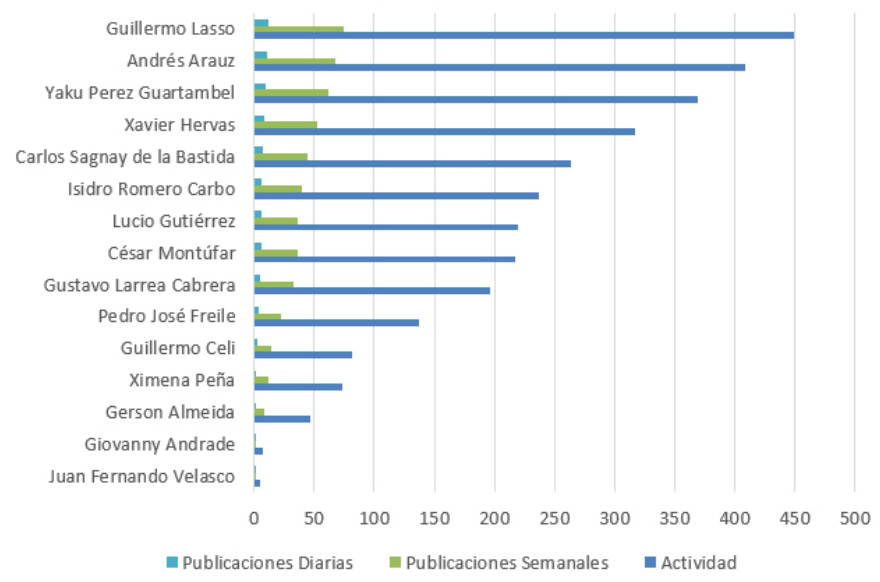

Gráfico 4: Actividad en Facebook

Fuente: Elaborado por los autores

\subsubsection{Nivel de servicio}

El nivel de servicio evalúa la respuesta de los candidatos a los seguidores. En el periodo analizado el nivel de servicio es 0 para todos los candidatos, demostrando que la interacción es baja, lo que refleja un bajo compromiso, principalmente, de Arauz que registra 522 publicaciones de sus fans, y Lasso que tiene 168 publicaciones sin contestar.

\subsubsection{Participación}

Se observa, que los ecuatorianos son poco activos en los perfiles de los políticos, en el periodo, de un poco más, de tres meses se registran 11.954.955 interacciones cuando existen 13.3 millones de usuarios de la red (Del Alcázar Ponce, 2021). Existe una relación directa con los candidatos más activos en la red y los que consiguen mayor participación de los seguidores, sin embargo, la calidad o contenido publicado puede marcar la diferencia, Arauz y Hervas con menor número de publicaciones que Lasso y Pérez los superan en interacción, y se debe resaltar la participación de los seguidores de Pedro José Freire que, con pocas publicaciones, consigue una importante interacción de sus fans

Los electores ecuatorianos son poco comprometidos en las cuentas de FB, el $72 \%$ de las interacciones corresponde a reacciones, y en igual proporción (14\%) los usuarios comentan o comparten información, generando poca viralidad de las propuestas electorales. Se precisa aclarar que, los ciudadanos presentan mayoritariamente actitudes positivas al reaccionar en las cuentas de los candidatos presidenciales. El 74\% de las reacciones corresponden a la opción "me gusta" y el 15.8\% de los seguidores seleccionan la opción "me encanta", únicamente el 1.2\% de los usuarios dan clic en la reacción "me enoja" y el $0.2 \%$ "en me entristece". El candidato que recibe mayor número de reacciones negativas es Guillermo Lasso (49.186), seguido de Andrés Arauz (39.457), Xavier Hervas (6.559) y Yaku Pérez (6.479), pero también son quienes obtienen el mayor porcentaje de reacciones positivas.

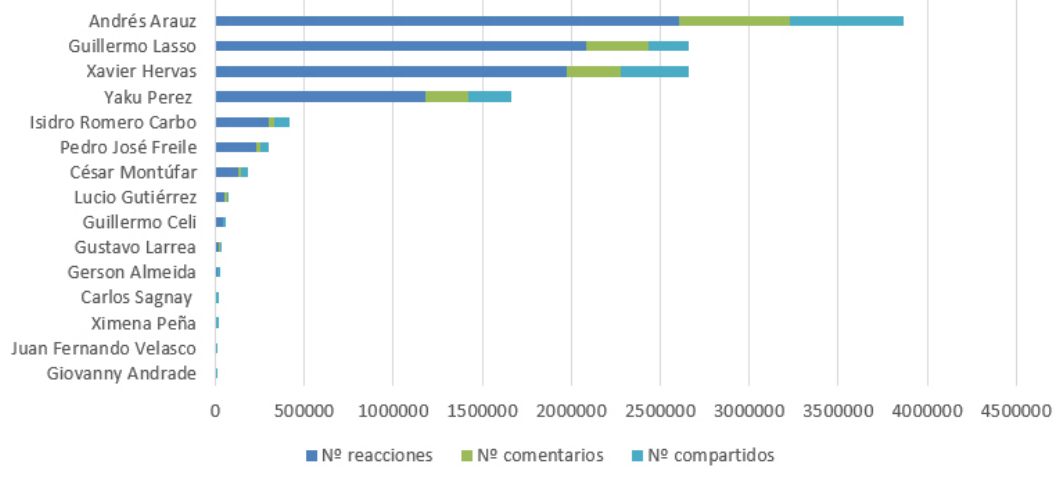

Gráfico 5. Participación de los fans en Facebook

Fuente: Elaborado por los autores 
En el caso de los candidatos a la presidencia, el análisis demostró que el engagement es proporcional a la participación de los candidatos en la red; Arauz, Hervas, Lasso y Pérez son quienes consiguen mayor compromiso de sus seguidores, pero se debe recalcar, el alto porcentaje de engagement de Freire, que con contenidos muy claros y de valor logra que sus seguidores participen más activamente, demostrando mayor compromiso.

\subsection{6. Índice de la comunicación 2.0}

El modelo de evaluación de la comunicación 2.0 es cuantitativo, con la finalidad de tener una visión exacta del proceso de comunicación interactiva. Para la valoración se pondera las acciones, reacciones e interacciones, tanto de los candidatos como de los electores 2.0, en las cuentas de Facebook, lo que permite crear los índices de presencia, crecimiento, actividad, nivel de servicio, participación y engagement, variables seleccionadas en función a las características básicas de la comunicación 2.0. Estos índices, obtenidos en el proceso de investigación, admiten valorar y catalogar el desempeño de los candidatos presidenciales y de cada variable estudiada.

El Modelo permite evaluar la comunicación 2.0 desde una perspectiva comparativa, es decir interrelaciona varias cuentas de un determinado medio social. Una vez que se obtienen los KPIs para evaluar las variables seleccionadas se procede a normalizar los datos, normalizar los indicadores de desempeño permite crear una escala estándar que guarda las mismas proporciones entre las variables, en este proceso se aplica la fórmula: $\mathrm{Ni}=(\mathrm{X}-\mathrm{Min} / \mathrm{Max} .-\mathrm{Min}) *$.100 . Finalmente, estos resultados se interrelacionan para elaborar el índice de la comunicación 2.0. Una vez que se tiene los índices, por cada una de las cuentas, se suman los resultados de las variables analizadas y se divide para seis con el objetivo de ponderar los resultados y catalogarlos (Altamirano et al, 2018).

Tabla 1. Índice de la comunicación 2.0

\begin{tabular}{|c|c|c|c|c|c|c|c|c|}
\hline Candidatos & $\begin{array}{l}\frac{\pi}{0} \\
0 \\
0 \\
0 \\
0 \\
0\end{array}$ & 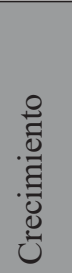 & 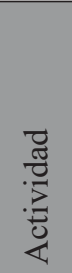 & $\frac{\frac{0}{0}}{\sum^{0}}$ & 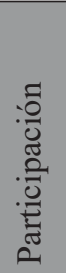 & 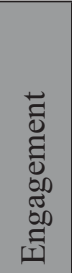 & 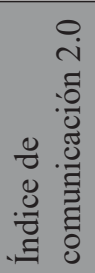 & 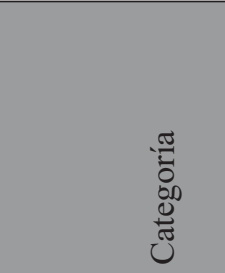 \\
\hline Guillermo Lasso & 100 & 19 & 100 & 0 & 69 & 63 & 58 & \multirow{4}{*}{ Aceptable } \\
\hline Andrés Arauz & 13 & 1 & 91 & 0 & 100 & 100 & 51 & \\
\hline Yaku Perez & 26 & 53 & 82 & 0 & 43 & 48 & 42 & \\
\hline Xavier Hervas & 14 & 1 & 70 & 0 & 69 & 89 & 41 & \\
\hline Pedro José Freile & 5 & 100 & 30 & 0 & 8 & 23 & 28 & \multirow{2}{*}{ Aceptable bajo } \\
\hline Isidro Romero Carbo & 2 & 41 & 52 & 0 & 11 & 19 & 21 & \\
\hline César Montúfar & 4 & 18 & 48 & 0 & 5 & 9 & 14 & \multirow{9}{*}{ Mejorable } \\
\hline Lucio Gutiérrez & 3 & 5 & 48 & 0 & 2 & 4 & 10 & \\
\hline Carlos Sagnay & 1 & 2 & 58 & 0 & 0 & 0 & 10 & \\
\hline Gustavo Larrea & 3 & 2 & 43 & 0 & 1 & 2 & 8 & \\
\hline Guillermo Celi & 3 & 10 & 17 & 0 & 1 & 7 & 6 & \\
\hline Juan Fernando Velasco & 27 & 3 & 0 & 0 & 0 & 5 & 6 & \\
\hline Ximena Peña & 1 & 3 & 15 & 0 & 0 & 1 & 3 & \\
\hline Gerson Almeida & 2 & 0 & 9 & 0 & 1 & 5 & 3 & \\
\hline Giovanny Andrade & 0 & 1 & 0 & 0 & 0 & 0 & 0 & \\
\hline
\end{tabular}

Fuente: Elaborado por los autores

Como se puede observar, no existe un proceso de comunicación en los fanpages de los candidatos a la presidencia de Ecuador, ninguno de los candidatos alcanza la categoría de óptimo y aceptable alto, se crean las redes sociales, sin un objetivo claro y se las utiliza, principalmente para difundir información, 
como un medio de comunicación tradicional, cuando su principal característica es la interactividad. Los contenidos que difunden, como se comprueba en la investigación, son pensados en los intereses del candidato, no en las necesidades de la audiencia, por esa razón, la participación y el compromiso de los electores son bajos. No puede ser coincidencia, que los cuatro candidatos con mayor aceptación popular y votación sean quienes obtienen mejor puntaje en el proceso de comunicación, esto se debe, principalmente, a que logran construir una comunidad, mantienen una actitud 2.0, publican frecuentemente en sus cuentas sociales, a pesar que el nivel de respuesta e interacción es bajo.

Al evaluar los procesos de comunicación 2.0 entre los candidatos a presidentes y los electores $(\mathrm{O} 1)$ se concluye que Facebook es subutilizado como un medio tradicional que no fomenta la participación (Gamir-Ríos, 2021), es decir, los candidatos presidenciales, únicamente, difunden información y carecen de espacios que promuevan el debate o diálogo con los electores, "pues son casi nulas las intenciones de los actores políticos por interactuar con los usuarios y viceversa; por lo que las redes sociodigitales son empleadas como una especie de agenda política y medio de difusión de sus campañas de marketing político" (Toxtle y González, 2020. p. 89), por lo que se concluye, que en las cuentas de los candidatos presidenciales no se promueve una comunicación 2.0, interactiva y participativa, sino que se emplea como plataforma de broadcasting (Recalde, Baquerizo y Zunino, 2019; Neira, Uribe y Gonzalez, 2020). En ninguna de las cuentas analizadas se encuentran publicaciones que promuevan el diálogo y la participación ciudadana o que permitan al candidato escuchar y conocer las necesidades de sus electores, tampoco se realizan transmisiones en vivo para interactuar con el ciudadano, mucho menos se identifican espacios interactivos, como foros, encuestas, entre otras alternativas que brinda la red social.

\section{Análisis de los datos con el software de procesamiento de lenguaje natural (LIWC)}

Un elemento de la estrategia política en contexto de campaña es la emocionalidad del discurso de los candidatos, para ello se procesan los datos con el software LIWC y en los resultados se observa que el lenguaje que emplean los candidatos hacia sus seguidores es directo, utilizan mayormente los pronombres "él/ella" en sus publicaciones (ver Gráfico 6), tal como se observa en las siguientes publicaciones:

La paz y tranquilidad social se basa en la confianza a la institucionalidad. Un proceso electoral honesto es lo que demanda el pueblo ecuatoriano. \#AbramosLasUrnasEcuador \#EstamosConYaku

Por años, miles de jóvenes no pudieron entrar a la universidad o se les negó estudiar la carrera de su preferencia. Con \#CapacidadParaCambiar, tendrán la libertad de escoger su propio camino para crecer. ¡Vamos a dar autonomía a las universidades! \#LassoPresidente2021. (Lasso, 2021)

También se evidencia que Aráuz y Hervas son los únicos que, al dirigirse a sus seguidores, emplean el pronombre "nosotros", característica populista que "abarca al orador, a su partido, a sus partidarios y al pueblo" (Charaudeau, P., y Gentile, A., 2009, p. 271). El uso de la primera persona del plural connota una relación con el colectivo al que se dirige y también determina cierta autoridad en el que habla (Ruas el al., 2013), tal como se advierte en los siguientes posts:

Esta segunda vuelta debe ser un ámbito de discusión democrática, que enriquezca a nuestra sociedad. Nosotros seguiremos recorriendo, escuchando y brindando propuestas. Planteamos la unidad del amor por nuestro país, del amor por el pueblo, con un objetivo claro: un futuro mejor para el Ecuador. (Âráuz, 2021)

La encuesta de https://www.atlasintel.org/ nos ubica en 4to lugar con 3,3\% de intención de voto. Crecemos! Gracias a todos quienes apuestan por nosotros, la \#GenteNueva. Comparte nuestras propuestas, ayuda a que más gente se sume. El compromiso es hoy. \#Atrévete. \#XavierHervasPresidente. (Hervas, 2021) 
USO DE PRONOMBRES

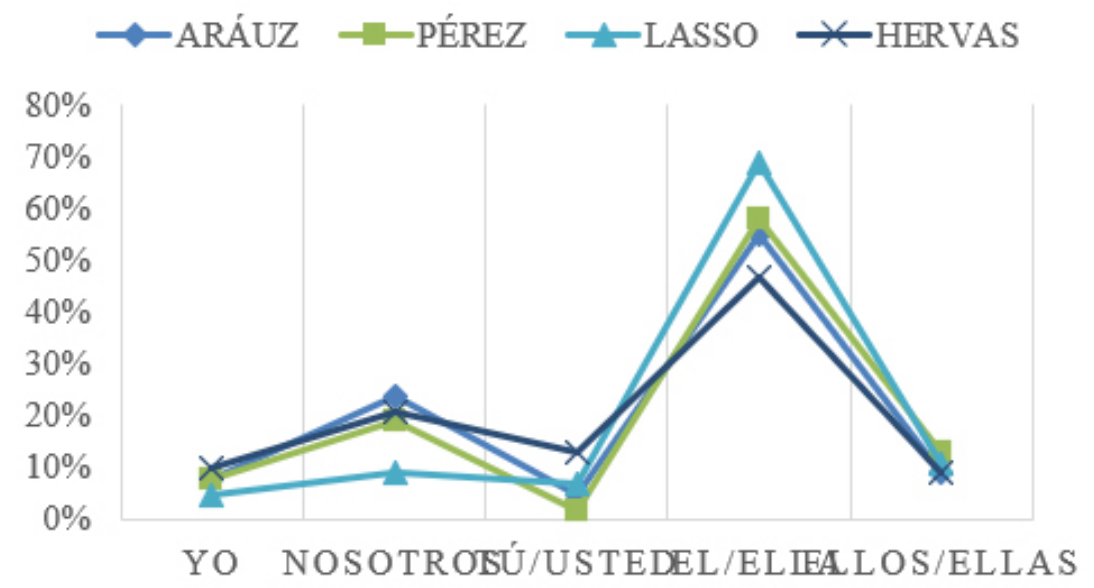

Gráfico 6: Frecuencia de uso de pronombres en los posts de los candidatos políticos

Fuente: Elaborado por los autores

En cuanto al uso de los pronombres, se observa que, como ya se dijo, el discurso de los candidatos no es de tipo populista, porque "no se configura un nosotros y un ellos, es decir, por un lado, un líder y sus seguidores; por el otro, los enemigos de ese líder, que también son enemigos del pueblo" (Patriau, 2012 , p. 300). En el uso del lenguaje de los candidatos analizados se evidencia, en mayor medida, el uso del pronombre personal "él/ella" en sus publicaciones y los temas que aparecieron con mayor frecuencia fueron logro y trabajo, un discurso "coherente" en un país azotado por la crisis económica mundial post pandemia.

Además, el análisis permite confirmar que en el discurso de campaña todos emplean un lenguaje positivo (ver Gráfico 7). Esto condice con el "carisma" como característica propia del líder político. Las palabras más utilizadas en los posts son: próspero, esperanza, orgullo, sonrisa, cariño, energía, motivación, entusiasmo, alegría, honestidad, feliz, buena vibra, eficiente, mejores, oportunidades, entre otras. Estas se observan acompañadas de hashtags con los eslóganes de campaña. A continuación, se anotan dos publicaciones que hacen referencia a la emocionalidad positiva:

Gracias Manabí con sus playas hermosas y un pueblo hospitalario que nos convidó tanta alegría en un mágico atardecer, disfrutando de donde venimos: el \#Mar y sus \#Aguas. ¡Juyayay \#Manabi! \#YakuPresidente \#ClaroQueSePuede \#YakuEs. (Yaku, 2021)

Los invito a votar por el CAMBIO y un Plan de Gobierno que busca la libertad, el fortalecimiento de la democracia y construir un país de oportunidades. Con alegría y entusiasmo, este 7 de febrero vamos a votar por la \#CapacidadParaCambiar. LASSO PRESIDENTE 202. (Lasso, 2021)

También se observa que, en menor medida, se utiliza lenguaje con emocionalidad negativa. Sin embargo, dentro de esta categoría se distinguen más palabras asociadas a la categoría "enfado" (85\%) que a "triste" (15\%). (15\%). De esto se infiere que, en un contexto electoral, el discurso se desarrolle en torno a elementos como disputas y descalificación entre los candidatos, rechazo al gobierno saliente, denuncias por irregularidades en el proceso electoral o discusiones por asuntos políticamente relevantes (ver Gráfico 7). En el elenco están: lucha, corrupción, indignación, violencia, fraude, robar, violencia de género, engañar, calumnias, inseguridad, desempleo, pobreza, delincuente, crimen, asesinos, represión, protesta, entre otras. 


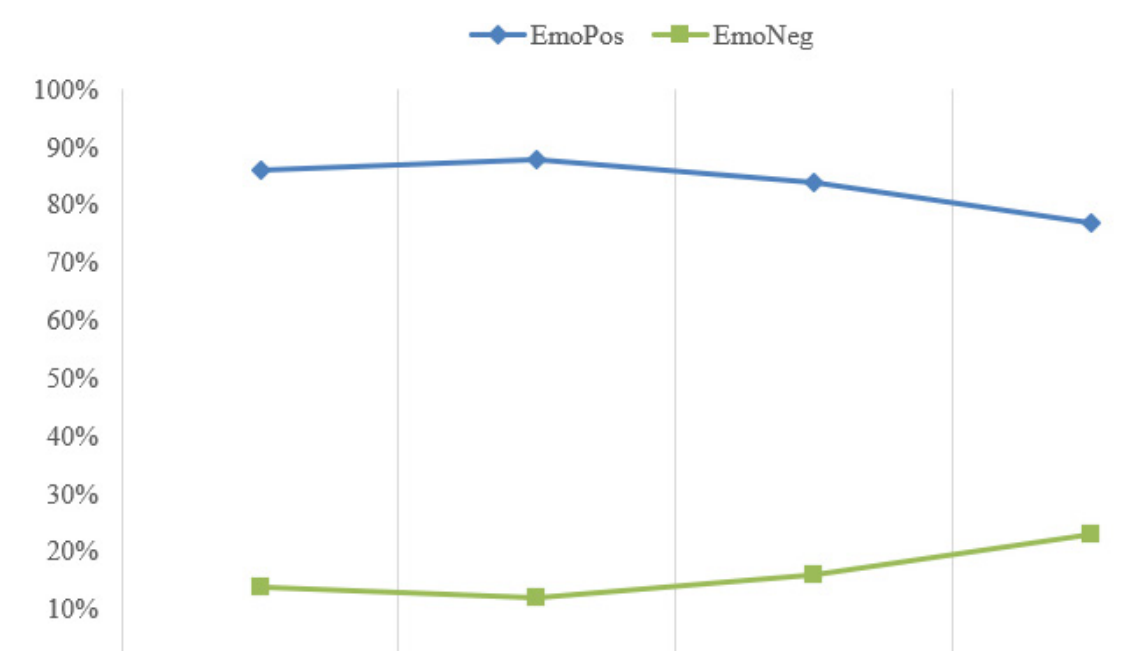

Gráfico 7: Uso de emociones positivas y negativas en las publicaciones de los candidatos

Fuente: Elaborado por los autores

Publicaciones como: "No permitas que nos traten como estúpidos. Estamos peleando contra la demagogia, contra la corrupción. \#DebatePresidencial2021 \#EcuadorDecide2021 \#XavierHervasPresidente (Hervas, 2021)", representan el uso de connotaciones negativas en el discurso de campaña de los candidatos.

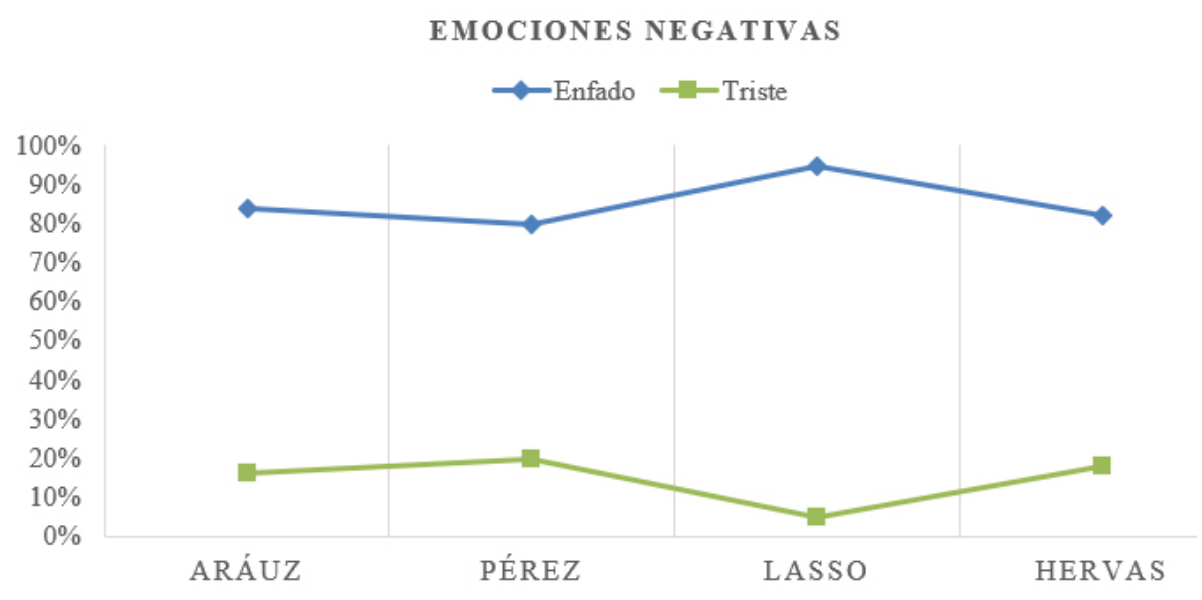

Gráfico 8: Uso de emociones negativas en las publicaciones de los candidatos

Fuente: Elaborado por los autores

A partir del análisis del uso del lenguaje de los candidatos se identifican otras palabras asociadas a temas como logro y trabajo. Hervas es el que más emplea palabras asociadas a la categoría dinero en la mayoría de sus publicaciones (54\%), esto se conjuga con sus propuestas de campaña en relación a los planes de reactivación para el sector agropecuario y el acceso a mercados nacionales e internacionales. En cambio, Lasso utiliza un lenguaje relacionado a logro (41\%) y trabajo (42\%), seguido por Pérez (logro $28 \%$ y trabajo $37 \%$ ) y Aráuz (logro $29 \%$ y trabajo $30 \%$ ).

El primero está relacionado con la oferta de crear dos millones de empleos en los cuatro años de gobierno; el segundo ofrece trabajo, futuro y dignidad; y, el tercero, trabajo digno. Se muestran algunos ejemplos:

En nuestros 4 años de gobierno CREAREMOS 2 MILLONES DE EMPLEOS. Los ecuatorianos tendrán un trabajo digno y productivo, bien pagado y con seguridad social. \#ConLassoHayEMPLEO \#PropuestasDeLasso \#CapacidadParaCambiar \#LassoPresidente2021. (Lasso, 2021)

\#EnVivo | Gracias al pueblo de Pastaza por su entusiasmo y alegría. ¡Falta cada vez menos! Con la 
esperanza renovada, se viene el Ecuador del trabajo, el futuro y la dignidad. (Aráuz, 2021)

Pérez es el único candidato que aborda el tema relacionado al hogar (16\%). Pocas son las referencias vinculadas a religión (ver Gráfico 9).

¿Que estoy viajando a México? Sí, estoy en la calle México y Remigio Crespo de mi linda ciudad de los ríos de Cuenca. Jugando en familia con agüita, mishkitullito y una alegre música nacional. Juyayay el \#Carnaval2021 (Pérez, 2021)

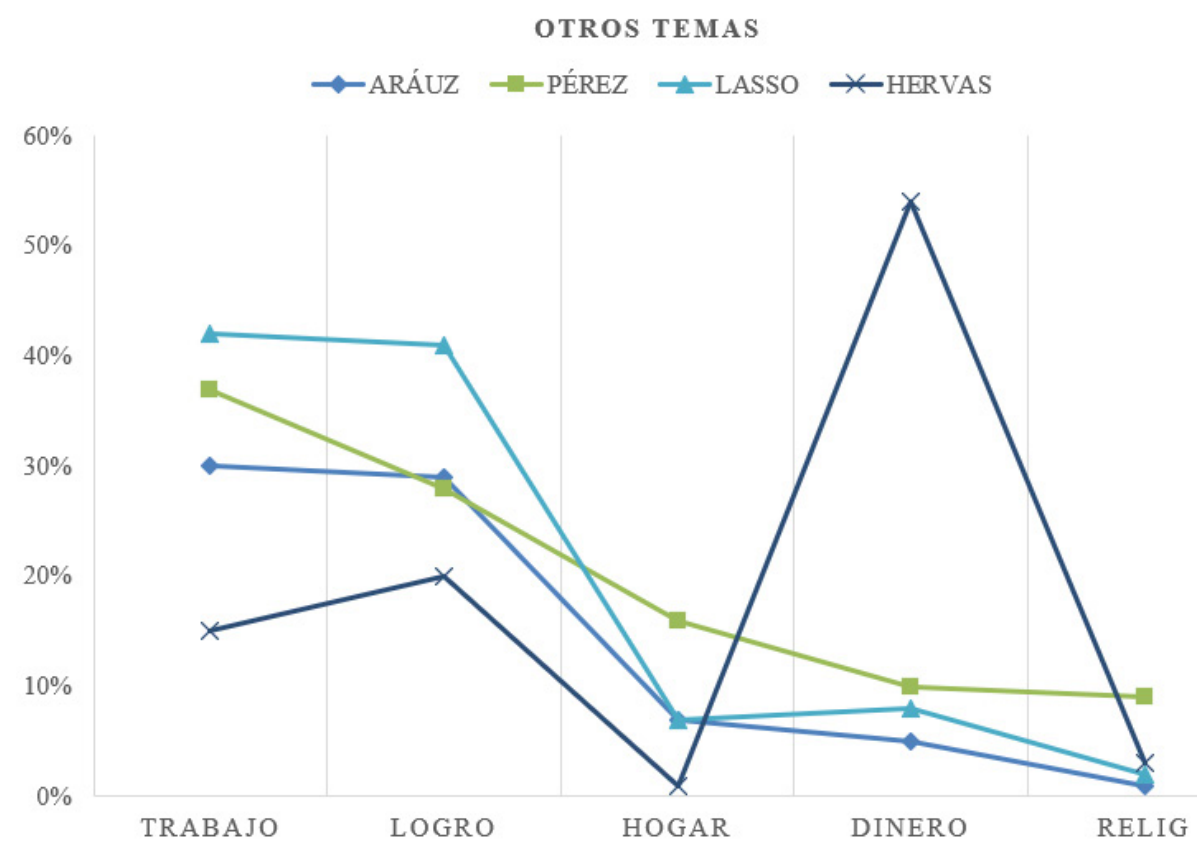

Gráfico 9: Temas identificados con la herramienta LIWC en las publicaciones de los candidatos

Fuente: Elaborado por los autores

Del análisis emocional del uso del lenguaje de los candidatos en campaña política se puede concluir que, en este caso -la emoción positiva- no es un elemento que influye en el aumento de interacción entre los postulantes y sus seguidores. Esto responde al hallazgo de Stieglitz y Dang-Xuan (2012) que sostienen que, los mensajes negativos publicados en el muro de Facebook reciben más comentarios y generan mayor interacción que los positivos. Asimismo, estos autores determinan que existe una mayor interacción porque la gente "comenta más en discusiones sobre problemas, asuntos o preocupaciones políticamente relevantes" (p.12).

\section{Análisis de la agenda política}

Tomando en cuenta el porcentaje de atención, se obtienen los siguientes hallazgos: Los cuatro candidatos publican, mayoritariamente, contenido retórico, emocional, o la transmisión de eventos de campaña (código 0) que no representan las políticas propuestas por los partidos, coincidiendo con los estudios realizados por López-Meri et al. (2020) que afirman que "los partidos y sus líderes tienden a promocionar sus actos de campaña y sus programas, así como a pedir el voto, pero apenas utilizan Facebook para incentivar el diálogo real con sus seguidores" (p.241).

En menor proporción los candidatos presentan su oferta electoral: Lasso basa su propuesta en trabajo y salud; Arauz habla de gobierno y administración pública y educación. Por su parte Hervas, aborda las temáticas referentes a gobierno y administración pública con economí; y Pérez se enfoca en los códigos de gobierno y administración pública y ambiente. 
Tabla 2. Porcentaje de atención de los posts de Facebook de los 4 candidatos

\begin{tabular}{|c|c|c|c|c|}
\hline Código & Guillermo Lasso & Andrés Arauz & Xavier Hervas & Yaku Pérez \\
\hline Retórica & 72,61 & 79,04 & 54,55 & 54,07 \\
\hline Economía & 2,59 & 1,72 & 1,97 & 2,22 \\
\hline Derechos & 0,27 & 1,37 & 0,98 & 2,47 \\
\hline Salud & 3,48 & 1,37 & 1,97 & 0,74 \\
\hline Agricultura & 0,45 & 0,69 & 0,74 & 0,74 \\
\hline Trabajo & 8,21 & 1,49 & 0,25 & 0,25 \\
\hline Educación & 1,07 & 1,83 & 1,47 & 3,70 \\
\hline Ambiente & & 0,11 & 0,25 & 5,43 \\
\hline Transporte & 0,36 & 0,80 & 0,49 & 0,99 \\
\hline Política Interior & 2,85 & 0,92 & 0,49 & 0,74 \\
\hline Política Social & 1,43 & 0,80 & & \\
\hline Política Comercial & 1,07 & 1,49 & & 0,25 \\
\hline Defensa & 0,18 & & & \\
\hline Investigación & 0,09 & 1,37 & 0,25 & 0,49 \\
\hline Comercio Exterior & 1,07 & & & \\
\hline Política exterior & & & 0,49 & \\
\hline Gobierno & 2,41 & 6,87 & 36,12 & 27,90 \\
\hline Recursos Naturales & 1,87 & & & \\
\hline Eventos culturales & & 0,11 & & \\
\hline
\end{tabular}

Fuente. Elaborado por los autores.

Lo más importante a destacar es que, el porcentaje de atención coincide parcialmente con la lógica partidista de propiedad de temas, de esta manera, si se toma en cuenta la concentración de atención en gobierno y administración pública, tema que se puede considerar propio de partidos situados en una posición ideológica de izquierda, coincide con los candidatos Arauz, Pérez y Hervas, lo cual se relaciona con lo que determina la literatura académica. En el caso de Lasso, no existe un paralelismo ideológico, es decir, considerando que es un candidato en el espectro ideológico de derecha, este no se enfoca en los temas conservadores que la literatura académica determina, por ejemplo, economía o política interior y justicia, de hecho, se enfoca en temas como trabajo y salud. Esto puede ser justamente porque la agenda pública y las encuestas de opinión pública, determinaron cuales son las temáticas más importantes para los ecuatorianos donde se evidencian temas como trabajo (dentro de economía) y salud como las más importantes. Cuestiones particulares de cada candidato, se ve que van de la mano con los planes de trabajo de los candidatos. Por ejemplo, Pérez tiene como tercer tema más importante en atención, la problemática del ambiente. Hervas y Arauz, economía y educación respectivamente.

Finalmente, el tema que más llama la atención, es que la concentración de la agenda política no va a temas con contenido político, más bien todo lo contrario, se enfoca en temas que, por el propósito de este estudio, hemos llamado "retórica" donde se evidencia que los candidatos usan eslóganes de campaña, narrativas discursivas, lenguaje emocional sin fondo político. Esto muestra, la necesidad de transmitir un mensaje de forma, de persuasión, sin propuestas reales pero que generan resonancia cultural, más no de señalar puntualmente las propuestas de campaña y sus formas de consecución. Ello se evidencia en el gráfico 10. 


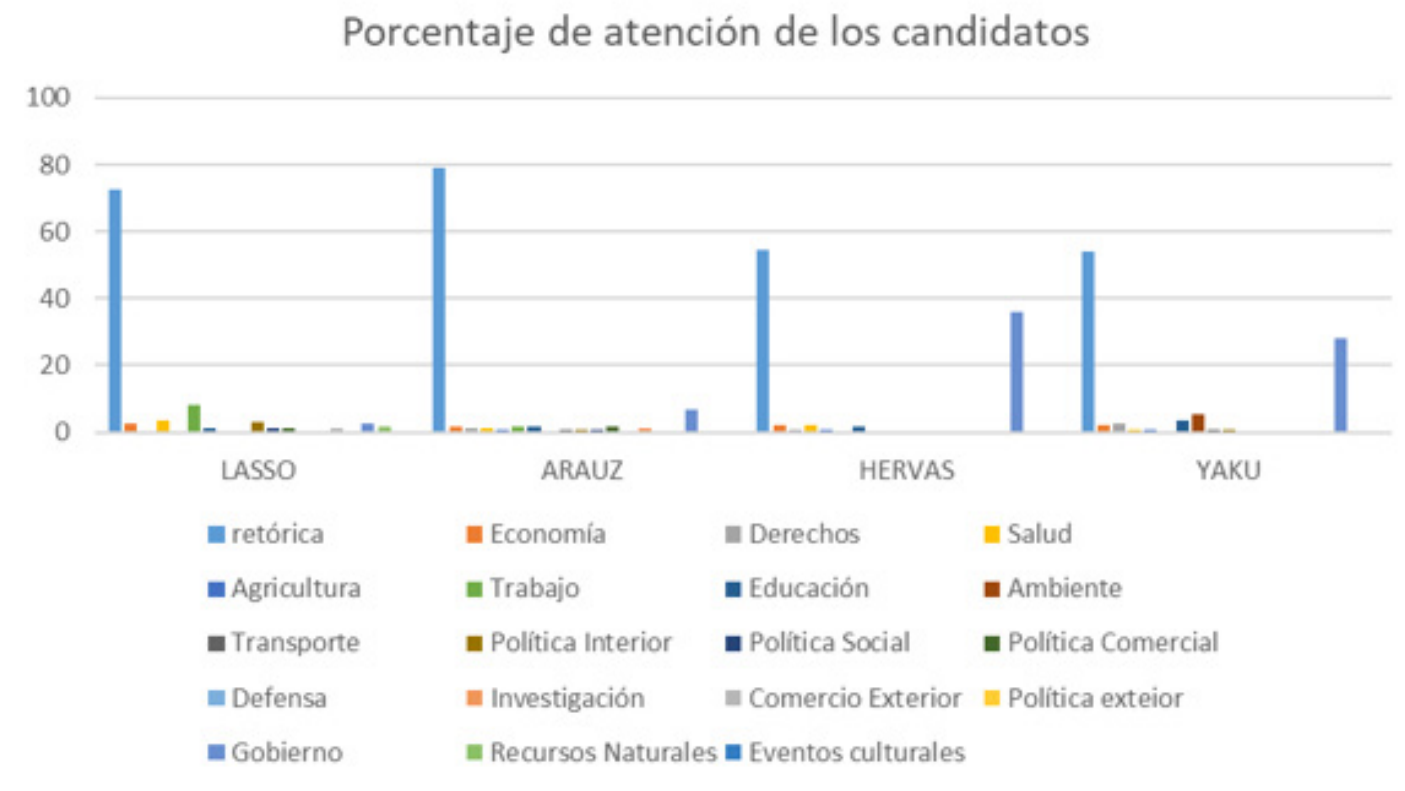

Gráfico 10: Porcentaje de atención de los posts de Facebook de los cuatro candidatos

Fuente: Elaborado por los autores

\section{Conclusiones}

Mucho se habla de la importancia de la red social FB y su impacto en la campaña electoral, se la considera como una plataforma de difusión de las ofertas políticas que permite llegar a una audiencia segmentada, pero numerosa, y algunos estrategas más idealistas hablan de la posibilidad de establecer relaciones entre candidatos y electores. Sin embargo, la realidad es completamente diferente, la investigación demuestra que, en Ecuador, el impacto es realmente bajo, únicamente, el 15.9\% de los usuarios de FB del país siguen la cuenta de un candidato presidencial, probablemente, por la falta de estrategia en las campañas y la difusión "improvisada" de contenidos que no son interesantes para la audiencia porque no se adaptan a sus necesidades o intereses y mucho menos presentan soluciones a sus problemas.

Por su parte los electores, a falta de una estrategia creativa, contenidos interesantes y espacios que promuevan el debate adoptan una actitud pasiva, pues su interacción es poco comprometida, participan a través de la opción "me gusta" y no generan ni comparten contenidos, acciones indispensables para obtener referenciación y viralidad, lo que permiten que más personas se sumen a la propuesta electoral. A pesar de estos resultados, existe una coincidencia, los cuatro candidatos que mejor desarrollan la estrategia de comunicación digital son quienes lideran el proceso electoral, aunque se debe recalcar que también son quienes pagan mayor pauta publicitaria. En la red social Facebook, Lasso invirtió 598.410 dólares, Arauz pautó 210.699 dólares, Pérez pagó 180.148 dólares en anuncios y Hervas 70.687 dólares (Facebook, 2021).

Entre los resultados se destaca, que la participación de los electores es proporcional a la actividad en la cuenta, cuando los niveles de publicación son bajos, es decir, a menor número de publicaciones de los candidatos es menor la interacción de los ciudadanos, pero, se debe resaltar que la interactividad está influenciada por la calidad de los contenidos publicados y el discurso político. Otro hallazgo interesante de la investigación es que las comunidades más pequeñas generan mayor engagement, a diferencia de cuentas con mayor número de seguidores, puede ser motivo de otra investigación verificar la autenticidad de los perfiles.

Como resultado del análisis de los elementos lingüísticos (O2), se identificó que el uso del lenguaje no responde a un discurso populista y que la emocionalidad de las publicaciones es de tipo positiva, esto determina que el mensaje utilizado por los líderes políticos es una estrategia que permite acelerar la difusión del mensaje en la red (Dang-Xuan et al, 2013) y genera un incremento de votos (Zullow et al,1988). Para futuras investigaciones es importante considerar que una limitación de la herramienta 
de procesamiento de lenguaje natural (LIWC), es que no discrimina el uso de los hashtags en las publicaciones.

Finalmente, al analizar la agenda política digital y cuáles fueron los temas que abordaron los candidatos en la red social (O3), se determina que la agenda política usada en redes sociales tiene netamente un enfoque retórico o en su mayoría se enfoca en ello, dejando para el futuro la necesidad de ahondar esto y posiblemente con su relación discursiva con el populismo.

\section{Referencias}

Aguirre, P. R. (2016). The Language approach of Legitimation of the Presidents of the 21st Century Socialism: A new perspective of autopoiesis. [Tesis de maestría, Erasmsus University of Rotterdam]. https://thesis.eur.nl/pub/37316

Aguirre, P. R. (2020). Case study: what did Rafael Correa say? Comparing political attention across 10 years of the Ecuadorian president's discourses. Revista de Administração Pública, 54, 1546-1564.

Alashri, S., Srivatsav Kandala, S., Bajaj, V., Parriott, E., Awazu, Y., y C Desouza, K. (3 - 6 January, 2018,). The 2016 US Presidential Election on Facebook: an exploratory analysis of sentiments. 51st Hawaii International Conference on System Sciences, Hawaii, USA.

Alonso Mosquera, M. y Muñoz de Luna, Á. (2014). Redes sociales y consumidores: participación y construcción de imagen. Revista Historia y Comunicación Social 19 (Esp.), 211 - 221.

https://doi.org/10.5209/rev_HICS.2014.v19.45022

Altamirano Benítez, V., Ochoa, S. y Túñez, M. (13 - 16 de junio de 2018) Models to assess communication 2.0 on websites and organizational social media. 13th Iberian Conference on Information Systems and Technologies (CISTI), Badajoz, España.

Ruas, J., Fernández, M. y Puentes, I. (2013). Aplicación de la herramienta LIWC al análisis del discurso político: Los mítines de los candidatos en las elecciones al Parlamento de Galicia de 2012. M. Mariño, T. González, y M. Pacheco (Eds.), En Investigar la Comunicación Hoy. Revisión de políticas científicas y aportaciones metodológicas (Vol.1 pp. 47-64). Facultad de Ciencias Sociales, Jurídicas y de la Comunicación.

Baquerizo-Neira, M. G., Almansa-Martínez, A., y Uribe, E. L. (2021). Proyección del discurso político en tiempos de campaña. ComHumanitas: revista científica de comunicación, 12(1), 32-51.

Barandiarán, X., Unceta, A. y Peña, S. (2020). Comunicación Política en tiempos de Nueva Cultura Política, Icono 14, 18 (1), 256-282. https://doi.org/10.7195/ri14.v18i1.1382

Baumgartner, F. R., y Jones, B. D. (2010). Agendas and instability in American politics. University of Chicago Press.

Blasco-Duatis, M., Coenders Gallart, G. y Sáez, M. (2018): Representación composicional de la intermedia agenda-setting de los principales grupos de medios y partidos políticos en las elecciones generales españolas de 2015. Revista Latina de Comunicación Social, 73, 264 - 292.

https://doi.org/10.4185/RLCS-2018-1255

Bode, L., Vraga, E. K., Borah, P., y Shah, D. V. (2014). A new space for political behavior: Political social networking and its democratic consequences. Journal of Computer-Mediated Communication, 19(3), 414-429.

Budge, I., y Farlie, D. (1983). Party competition: selective emphasis or direct confrontation?: an alternative view with data. SAGE Publications.

Budge, I., y Hofferbert, R. I. (1990). Mandates and policy outputs: US party platforms and federal expenditures. American Political Science Review, 84 (1), 111-131. 
Cogburn, D. L., y Espinoza-Vasquez, F. K. (2011). From networked nominee to networked nation: Examining the impact of Web 2.0 and social media on political participation and civic engagement in the 2008 Obama campaign. Journal of political marketing, 10 (1-2), 189-213.

Cruz, J., Carrillo, A. y Oñate, A. (2020). Coincidencia entre agenda mediática y política en las cuentas de Twitter de los 4 candidatos con más votos para la alcaldía de Quito. Marco, Revista de marketing y comunicación política, 6, 89-109.

Charaudeau, P. y Gentile, A. (2009). Reflexiones para el análisis del discurso populista. Discurso y Sociedad, 3(2), 253-279.

Chaves - Montero, A. (2018) La utilización de una metodología mixta en investigación social en K. Delgado, W. Gadea, S. Vera (Ed.), Rompiendo Barreras en la Investigación. Ediciones UTMACH.

Dang-Xuan, L., Stieglitz, S., Wladarsch, J. y Neuberger, C. (2013). An investigation of influentials and the role of sentiment in political communication on Twitter during election periods. Information, communication y society, 16(5), 795-825.

Del Alcázar Ponce, J. (Enero de 2021). Ecuador. Estado Digital Ene/21. Mentinno. https://www.mentinno.com/estadodigitalecuador/\#descarga

Deltell, L., Claes, F. y Osteso, J. M. (2013). Predicción de tendencia política por Twitter: Elecciones Andaluzas 2012. Ámbitos. Revista internacional de comunicación, (22), 91-100.

Dutt, R., Deb, A. y Ferrara, E. (11 - 14 December, 2018). "Senator, We Sell Ads": Analysis of the 2016 Russian Facebook Ads Campaign. International conference on intelligent information technologies, Chennai, India

Galeano, S. (29 de enero de 2021). Cuáles son las redes sociales con más usuarios del mundo (2021). marketing4ecommerce.net.

https://marketing4ecommerce.net/cuales-redes-sociales-con-mas-usuarios-mundo-ranking/

Gamir-Ríos, J. (2021). El uso político de Facebook en la campaña de las Elecciones Locales de 2015 en la ciudad de València. Dígitos. Revista de Comunicación Digital, 7: 211-227.

https://doi.org/10.7203/rd.vli7.218

Green-Pedersen, C. y Mortensen, P. B. (2010). Who sets the agenda and who responds to it in the Danish parliament? A new model of issue competition and agenda-setting. European Journal of Political Research, 49(2), 257-281.

Green, J. y Jennings, W. (7-9 September, 2012). Valence and Government Priorities: How issue ownership and issue salience shape US and UK policy agendas. In presentation at the Elections, Public Opinion and Parties (EPOP) Conference, Oxford, United Kingdom,.

Green, J. y Jennings, W. (2019). Party reputations and policy priorities: How issue ownership shapes executive and legislative agendas. British Journal of Political Science, 49(2), 443-466.

Jennings, W., Bevan, S. y John, P. (2011). The agenda of British government: The speech from the throne, 1911-2008. Political Studies,59 (1), 74-98.

Klingemann, H. D., Hofferbert, R. I. y Budge, I. (1994). Parties, policies, and democracy. Westview Press

Kushin, M. J. y Yamamoto, M. (2013). Did social media really matter? College students' use of online media and political decision making in the 2008 election. In New media, campaigning and the 2008 Facebook election. Mass Communication and Society, 13(5), 608-630, https://doi.org/10.1080/15205436.2010.516863 
López-López, P.-C., y Vásquez-González, J. (2018). Agenda temática y Twitter: elecciones presidenciales en América Latina durante el período 2015-2017. Profesional de la Información, 27(6), 12041214. https://doi.org/10.3145/epi.2018.nov.04

López-Meri, A.; Marcos-García, S. y Casero-Ripollés, A. (2020). Estrategias comunicativas en Facebook: personalización y construcción de comunidad en las elecciones de 2016 en España. Doxa Comunicación, 30, pp. 229-248. https://doi.org/10.31921/doxacom.n30a12

Neira, G. B., Uribe, E. L. y Gonzalez, C. R. (2020). Doble minoría en tiempos electorales. El caso de Mae Montaño una mirada desde Twitter como plataforma de comunicación política. Revista Ibérica de Sistemas e Tecnologias de Informação, (26), 1-14.

Newman, M. L., Groom, C. J., Handelman, L. D. y Pennebaker, J. W. (2008). Gender differences in language use: An analysis of 14,000 text samples. Discourse processes, 45(3), 211-236.

Ñopo, K. (2021). El ejercicio de la libertad de expresión en los procesos electorales a través de las redes sociales. Lawgic Tec - Revista de Derecho y Tecnología, 1, 94-103.

Pasek, J., Kenski, K., Romer, D. y Jamieson, K. H. (2006). America's youth and community engagement: How use of mass media is related to civic activity and political awareness in 14-to 22-year-olds. Communication Research, 33(3), 115-135.

Park, C., Shim, M., Eo, S., Lee, S. Seo, J., Moon, H. y Lim, H. (2021). Empirical Analysis of Korean Public AI Hub Parallel Corpora and in-depth Analysis using LIWC. arXiv:2110.15023.

Patriau, E. (2012). ¡ El populismo en campaña! Discursos televisivos en candidatos presidenciales de la Región Andina (2005-2006). Colombia Internacional, (76), 293-325.

Pennebaker, J. W., Mehl, M. R. y Niederhoffer, K. G. (2003). Psychological aspects of natural language use: Our words, our selves. Annual review of psychology, 54(1), 547-577.

Petrocik, J. R. (1996). Issue ownership in presidential elections, with a 1980 case study. American journal of political science, 825-850.

Pita, O., Baquerizo, G., Vaca, C., Mendieta, J., Villavicencio, M. y Rodríguez, J. (12 - 14 October, 2016). Linguistic profiles on microblogging platforms to characterize political leaders: the ecuadorian case on twitter. IEEE Ecuador Technical Chapters Meeting, Guayaquil, Ecuador.

Primicias. (21 de febrero 2021). Resultados primera vuelta electoral. Primicias. https://www.primicias.ec/noticias/politica/resultados-primera-vuelta-electoral/

Recalde, L., Baquerizo, G. y Zunino, E. (24 - 26 April, 2019). Women in Politics and Their Presence in Twitter: Argentina as a Case Study. Sixth International Conference on eDemocracy y eGovernment (ICEDEG), Quito, Ecuador.

Ramírez, M. G. y de Travesedo Rojas, R. G. (2021). Repensar la comunicación política en el contexto digital. Ciberdemocracia ¿democracia deliberativa?. Vivat Academia, 63-87.

Rodríguez Fidalgo, MI., Ruiz Paz, Y., Paino Ambrosio, A. y Jiménez Iglesias, L. (2017): El uso de Facebook como herramienta narrativa para articular el nuevo diálogo político. Estudio de caso de la II Asamblea Ciudadana de Podemos, "Vistalegre II". Revista Latina de Comunicación Social, 72, 849 860. https://doi.org/10.4185/RLCS-2017-1196

Stieglitz, S. y Dang-Xuan, L. (15 may, 2012). Impact and diffusion of sentiment in public communication on Facebook. European Conference on Information Systems ECIS 2012, Barcelona, España. 
Sweetser, K. D. y Lariscy, R. W. (2008). Candidates make good friends: Ananalysis of candidates' uses of Facebook. International journal of strategic communication, 2(3), 175-198. https://doi.org/10.1080/15531180802178687

Tausczik, Y. R. y Pennebaker, J. W. (2010). The psychological meaning of words: LIWC and computerized text analysis methods. Journal of language and social psychology, 29(1), 24-54.

Toxtle Tlachino, A. y Arnoldo, R. (2020) Facebook, una extensión de marketing y no un espacio deliberativo: Caso de Andrés Manuel López Obrador en elecciones presidenciales de México en 2018. Global Media Journal México,17 (33), 72-95.

Toro Jaramillo, I. y Parra Ramírez, R. (2006). Método y conocimiento. Metodología de la investigación. Editorial Universidad EAFI.T.

Túñez López, M. y Altamirano, V. (2015). La simetría interactiva de la comunicación organizacional. Revista DIRCOM, (108), $14-17$.

Valera-Ordaz, L., Calvo, D. y López-García G. (2018): Conversaciones políticas en Facebook. Explorando el papel de la homofilia en la argumentación y la interacción comunicativa. Revista Latina de Comunicación Social, 73, 55 - 73. https://doi.org/10.4185/RLCS-2018-1245

Van Steenburg, E. (2015). Areas of research in political advertising: a review and research agenda. International journal of advertising, 34(2), 195-231.

Vitak, J., Zube, P., Smock, A., Carr, C. T., Ellison, N. y Lampe, C. (2011). It's complicated: Facebook users' political participation in the 2008 election. CyberPsychology, behavior, and social networking, 14(3), 107-114.

Volkens, A. (2002). Manifesto Coding Instructions. Wissenschaftszentrum Berlina für Sozialforschung (WZB).

Williams, C. B. y Gulati, G. J. (2009). Explaining Facebook support in the 2008 congressional election cycle. Working Papers, 26.

Zullow, H. M., Oettingen, G., Peterson, C. y Seligman, M. E. (1988). Pessimistic explanatory style in the historical record: CAVing LBJ, presidential candidates, and East versus West Berlin. American psychologist, 43(9), 673.

\section{AUTOR/ES:}

\section{Verónica Altamirano - Benítez}

Universidad Técnica Particular de Loja

Doctora en Comunicación e Industrias Creativas con mención Internacional y Cum Laude por la Universidad Santiago de Compostela (España), Máster en Comunicación e Industrias Creativas por la Universidad Santiago de Compostela, Diplomada en Comunicación Organizacional, en Comunicación para el Desarrollo y Licenciada en Comunicación Social por la Universidad Técnica Particular de Loja (Ecuador).

Premio Extraordinario de Doctorado del Área de Ciencias Sociales y Jurídicas 2016 - 2017 de la Universidad Santiago de Compostela.

Coordinadora del Grupo de Investigación Gestión de la Comunicación Estratégica, coautora de libros internacionales, de artículos en revistas científicas, y divulgativas. Sus publicaciones incluyen temas relacionados a la Comunicación Organizacional, Comunicación Turística, Comunicación 2.0, Comunicación Digital, Comunicación Política y Comunicación para el Desarrollo.

vpaltamirano@utpl.edu.ec

Indice H: 11

Orcid ID: https://orcid.org/0000-0003-1238-1886

Google Scholar: goo.gl/QyrpfV 
ResearchGate: https:/www.researchgate.net/profile/Veronica-Altamirano-2

Scopus ID: $5 \underline{6891487500}$

Academia.edu: https://utpl.academia.edu/VAltamirano

Web of Science ID: AAZ-8572-2021

\section{Pablo Ruíz - Aguirre}

Universidad Internacional del Ecuador

Doctor $(\mathrm{C})$ en derecho y ciencia política por la u de Barcelona-España y Antwerp-Belgica. Máster en análisis político y asesoría institucional por la u de Barcelona-España. Máster en gobernanza por la universidad Erasmos de Róterdam Holanda. Especialidad en política pública y especialidad en gobierno local por el ISS de Holanda. Diplomado en Gestión pública por la U. Washington. Diplomado en Comunicación política y campañas electorales por la UIDE y U. Camilo José Cela (en proceso) Abogado por la UDLA. Director del Ecuadorian Policy Agendas Project. Sustemas de investigación son agenda política, discurso político. Es profesor de pregrado en la UIDE y de postgrado en la UTPL. paruizag@uide.edu.ec

Indice H: 1

Orcid ID: http://orcid.org/0000-0002-1504-7544

Google Scholar: https://scholar.google.com/citations?user=sJhLiBcAAAAJ\&hl=es

ResearchGate: https://www.researchgate.net/profile/Pablo-Ruiz-Aguirre

\section{Gabriela Baquerizo-Neira}

Universidad Casa Grande

Ph.D en Comunicación Social por la Universidad Austral de Buenos Aires (Argentina). Realizó su maestría en DIRCOM en Valencia-España. Sus principales temas de investigación son: comunicación política latinoamericana y redes sociales; análisis de sentimientos; reputación y comunicación estratégica. Directora del grupo de investigación "Comunicación, política y liderazgo en contextos sociales y digitales latinoamericanos". Coordinadora y autora de capítulos de los libros "Comunicación política en campaña. Identidad, imagen y lenguaje como elementos estratégicos en las campañas políticas latinoamericanas" y "Ciberdemocracia y ciudadanía". Actualmente es profesora e investigadora de la facultad de Comunicación de la Universidad Casa Grande.

gbaquerizo@casagrande.edu.ec

Indice H: 4

Orcid ID: https://orcid.org/0000-0002-4837-1076

Google Scholar: https://scholar.google.com/citations?user=PVauaEwAAAAJ\&hl=es\&oi=sra

ResearchGate: https://www.researchgate.net/profile/Gabriela-Baquerizo-3

Scopus ID: 57222629713

Web of Science ID: $\underline{\text { AAG-3414-2020 }}$ 
Anexo 1. Agenda política, 24 temas principales

\begin{tabular}{|l|l|l|l|}
\hline Código & Descripción & Código & Descripción \\
\hline $\mathbf{0}$ & Retórica & $\mathbf{1 4}$ & Vivienda \\
\hline $\mathbf{1}$ & Economía & $\mathbf{1 5}$ & Comercio y Banca \\
\hline $\mathbf{2}$ & Derechos & $\mathbf{1 6}$ & Defensa \\
\hline $\mathbf{3}$ & Salud & $\mathbf{1 7}$ & Investigación, tecnología y Comunicación \\
\hline $\mathbf{4}$ & Agricultura & $\mathbf{1 8}$ & Comercio exterior \\
\hline $\mathbf{5}$ & Trabajo & $\mathbf{1 9}$ & Política exterior \\
\hline $\mathbf{6}$ & Educación y Cultura & $\mathbf{2 0}$ & Gobierno \\
\hline $\mathbf{7}$ & Ambiente & $\mathbf{2 1}$ & Recursos naturales y gestión del agua \\
\hline $\mathbf{8}$ & Energía & $\mathbf{2 3}$ & Cultura \\
\hline $\mathbf{9}$ & Buen Vivir & $\mathbf{2 7}$ & Clima \\
\hline $\mathbf{1 0}$ & Transporte & $\mathbf{2 9}$ & Deportes \\
\hline $\mathbf{1 2}$ & Política Interior y Justicia & $\mathbf{3 0}$ & Obituarios y Sucesos mortales \\
\hline $\mathbf{1 3}$ & Política Social & & \\
\hline
\end{tabular}

Fuente: Elaborado por los autores 